\title{
Receivers Behavior in a ISDB-T's Single Frequency Network
}

Christian Fragoas Fernandez Rodrigues

Francisco de Assis Peres

Lisandro Lovisolo 


\title{
Receivers Behavior in a ISDB-T's Single Frequency Network
}

\author{
Christian Fragoas Fernandez Rodrigues, Francisco de Assis Peres, Lisandro Lovisolo
}

\begin{abstract}
Former television receivers were unable to demodulate signals under very adverse conditions. In order to mitigate some reception problems, the so-called multiple frequency networks were used for television broadcasting. The advance in broadcast technology to digital transmission, using Orthogonal Frequency Division Multiplexing, allows digital television broadcasting networks to employ a smaller number of frequency channels as compared to former analog networks. A common appealing case is the use of a single frequency channel for the whole digital broadcasting network, the so-called single frequency networks. These became an important requirement, as they allow the improvement of spectrum usage, since contiguous regions are covered using a smaller fraction of the spectrum as compared to the former existing broadcasting systems and considering the same quantity of broadcasting transmitters. The advances in digital television broadcasting and improvements in receivers mainly pushed this. Both allowed the increase in the receiver's capability to demodulate signals under adverse conditions. This work investigates some aspects of receiver's performance and behavior in single frequency networks. The tests encompass five digital television receptors. Using a laboratory setup inspired in the single frequency network's reception scenario, we present results of the performance of digital television commercial receivers. One assumes that signals from two transmitters arrive jointly in lineof-sight conditions at the receiver. Several settings in terms of power ratios between primary and secondary signals and their relative delays are evaluated. In comparison to data in previous works, one observes an improvement in the performance of receptors in terms of the required electric field strength for successful reception. The effect of the guard interval setup on the reception is also evaluated and reported. We also evaluate the loss in the reception margin, which is inherent to the deployment of single frequency networks. In addition, using a default reception model, we translate the power density values measured on laboratory to the minimal electrical field strength necessary for reception. The results show a general degradation in receptor's performance due to the $\mathrm{SFN}$ and we conclude that the specified minimum electric field strength needs a revision to accommodate these types of project.
\end{abstract}

Index Terms - Digital Broadcasting, Receiver's Performance, Single Frequency Networks, Orthogonal Frequency Division Multiplexing

\section{INTRODUCTION}

$\mathrm{W}$ ith the current shortage of the electromagnetic spectrum, broadcasters and regulators seek to optimize its use [1]. This is a requirement as the demands for frequency spectrum increase for the delivery of different and diverse communication services. An alternative to optimize the spectrum usage is to make each service provider use the same frequency band everywhere. For television delivery networks, this is known as a Single Frequency Network (SFN) and became a requirement for DTV (Digital Television) systems. In the traditional MFN (Multiple Frequency Networks), that are being shut down all around the globe, each transmitter employs a different broadcast channel in each coverage area; that is, in adjacent areas, the same channel is not used for transmission; this prevents strong interference at reception. In an SFN, spectrum is saved because multiple areas are covered using a single broadcast channel; the consequent optimization in spectrum easily follows.

MFNs were widely used in analog television coverage system design for large areas, mainly due to the limitations of analog receivers to avoid multipath propagation derived problems. For example, the basic plan developed by Anatel (Brazilian national telecommunications agency) [2] restricts the use of a single RF channel for each coverage area for analog television broadcaster. One of the main concerns in the development and standardization of some digital television systems was to provide a physical layer for the transmission and reception with the necessary tools for implementing SFN. That has made the deployment SFN DTV networks plausible. The shift from analog to digital data allows to implement some

Christian Fragoas Fernandez Rodrigues is TV Globo's Project Engineer and Masters' Student at the Universidade do Estado do Rio de Janeiro, Rio de Janeiro, RJ, Brazil (e-mail: christianfragoas@gmail.com.br).

Francisco de Assis Peres is Manager of the Department of Projects and Installations of Transmission Systems at TV Globo, Rio de Janeiro, RJ, Brazil (e-mail: francisco.peres@tvglobo.com.br).

Lisandro Lovisolo, DSc, is Professor of the Department of Eletronics and Telecommunications at the Universidade do Estado do Rio de Janeiro, Rio de Janeiro, RJ, Brazil (e-mail: lisandro@uerj.br).

transmission and reception improvements such as efficient channel coding schemes [2]. That is intended for improving 
reception fidelity and to make it possible to deploy SFNs in scenarios with interference leading to transmission errors within the channel coding correction capabilities. These are often given in terms of power ratios between the main and the secondary signals (interfering ones) and relative signal's delay among them. SFNs are each day more imposed, due to the save in bandwidth they provide. In this sense, their study is necessary in order to provide tools for realistic and reliable analysis and design of such networks.

Actual SFN technology allows to control multipath effects by means of transmitter synchronization [1,2->3,3->4]. That is, the broadcast signal by the different transmitters can be delayed in order to avoid significant inter-symbol interference at some coverage area, enabling demodulation of the OFDM signal. In areas where two or more signals arrive from different transmitting stations, signals must be kept within bounds so that the receiver is capable to deal with. That means that one should ensure that: 1) secondary signals have delays within the main signal guard interval setup; 2 ) if one or more secondary signals are received outside the predefined guard interval, then their relative power to the main signal must be such that they do not prevent correct recovery of OFDM symbols.

To make a DTV receiver to function properly it is necessary that: 1) it is not saturated by the incident signals; 2) it succeeds in demodulation, even under multipath. In the case of SFNs, one has control on the second effect since the transmitters can be synchronized.

With the development and evolution of the DTV, the use of SFNs for DTV broadcasting signal became plausible. The improvement in the receivers, which began to equalize more efficiently multipath impaired signals, allowed SFN DTV broadcasting.

This study aims to determine and analyze the threshold reception in line-of-sight conditions (for primary and secondary signals), which is the worst-case scenario for DTV broadcasting projects in a SFN network. This is accomplished through laboratory measurements, in scenarios that mimic those reception conditions. With this work, one aims at facilitating the design and deployment of SFN for DTV broadcasting.

The SFN'S design is not a trivial task. It is necessary to adjust the transmitters' power and network synchronization, this must consider all coverage area and transmitters but also all possible receivers at which signals from different broadcasting sites may arrive. This is important for the interference suffered by these receivers to be within acceptable bounds, i.e., not preventing the recovering of the data stream. [1]. That is, one must ensure that secondary signals are received with delays within the main signal's guard interval. On the other hand, if one or more secondary signals are received outside the guard interval, the relative power differences between the main signal and the secondary ones must be such that they do prevent the correct reception of the primary OFDM signal.

\section{TESTS SPECIFICATIONS AND SFN PERFORMANCE MEASURES}

The proposed experiments allow to analyze the combined effects of intersymbol interference and fading by multipath in an SFN network with two transmitters in line-of-sight with the receiver. Initially, we will examine the symbol constellation of the an OFDM carrier, in the presence of the two signals generated by the exciters. Then we analyze the performance of the receivers, their signal demodulation capability and the presence or not of blocks in the images reproduced by the televisions, with the presence of two signals and noise at their input. In both cases, different conditions were evaluated by varying the delay parameters between signals and the power difference between them.

The diagrams in Figure 1 and Figure 2 were designed in order to simulate different conditions of reception, relative delay and signal amplitudes that a received in an SFN may be subject to. This allows empirical measures and the evaluation of the receiver performance and constellation's behavior in different reception conditions. The transmission system settings used are presented in Table 1. It considers the SBTVD (Sistema Brasileiro de TV Digital). For more information on the parameters of SBTVD channelization and coding, the reader should refer to [2].

TABLE 1

DIGITAL SYSTEM CONFIGURATION USED IN THE EXPERIMENTS

\begin{tabular}{|c|c|}
\hline System & SBTVD \\
\hline Chanel & $29(560 \sim 566 \mathrm{MHz})$ \\
\hline Bandwidth & $6 \mathrm{Mhz}$ \\
\hline Layer B Modulation & $64 \mathrm{QAM}$ \\
\hline FEC (foward error correction) & $3 / 4$ \\
\hline Guard Interval & $1 / 8(126 \mu \mathrm{s})$ \\
\hline Sincronization Mode & Static Delay (offset) \\
\hline
\end{tabular}

\section{A. Symbol Reception-Constallation's Behavior Evaluation}

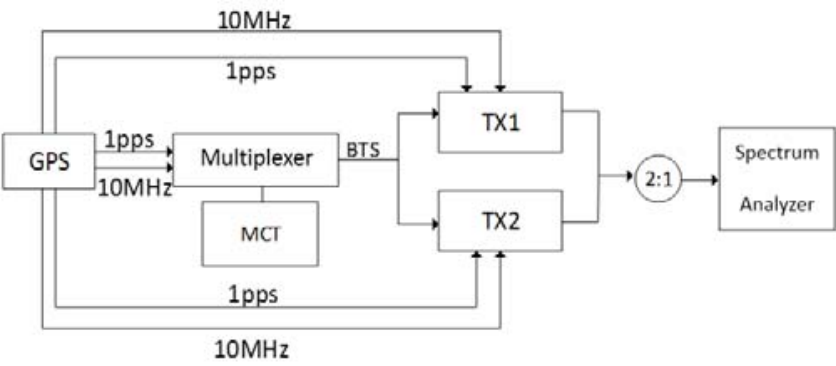

Figure 1. Laboratory setup employed to analyze symbol constellation at the receiver.

The laboratory setup (Figure 1) was designed to analyze the constellation behavior (symbol mapping) at the receiver, using a TV analyzer. The diagram consists in a multiplexer fed by the time and frequency network references 
(1pps, pulse per second, and $10 \mathrm{MHz}$ ) and by the ASI (Asynchronous Serial Interface) steam that comes from the Mux Controller (MCT). The broadcast transport stream is generated and will feed the exciters (Tx1 and Tx2) that generate channel $29 \mathrm{RF}$ signals (560 to $566 \mathrm{MHz}$ ).

The MCT is responsible for the network synchronism parameters. It composes the broadcast transport stream with the synchronism bits and the delay of each transmitter in the network.

The TV analyzer was employed to capture the constellation of the OFDM symbols. This was done varying power levels between the received signals to be $0,5,10,15$ and $20 \mathrm{~dB}$ and relative delay of 0 to $200 \mu \mathrm{s}$ in steps of $20 \mu \mathrm{s}$.

Section III presents the results and the concerning analyses of the receiver constellation behavior.

\section{B. Diagram 2 - Digital Television Comercial Receptors Behavior}

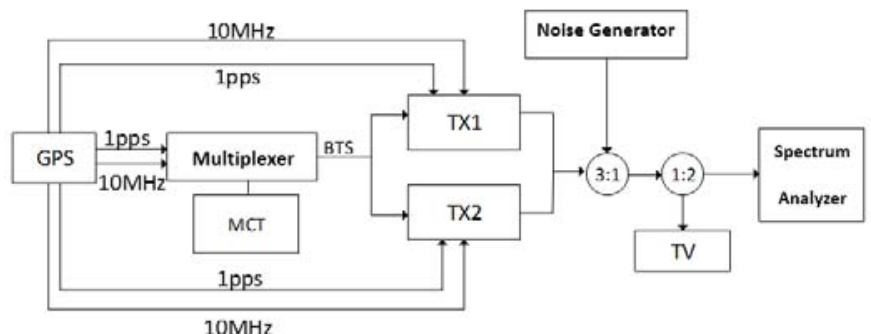

Figure 2. Laboratory setup used to analyze error in symbol reception, observed decoding errors in the displayed video point to the minimal signal to noise ratio (or signal to interference ratio) for correct signal decoding.

The diagram in Figure 2 is very similar to the one presented in Figure 1 with the addition of the noise generator plugged in the transmitter RF output to decrease its quality and the DTV receiver (i.e., a display and its built-in receiver) to decode the stream and present the decoded video and audio. This allows analyzing the behavior of the built-in receiver in conditions that are similar to a reception in SFN with 2 transmitters in line-of-sight with the receiver. Transmitters are configured as presented in Table 1. This allows to vary the noise power in order to obtain the required signal to noise ratio $(\mathrm{C} / \mathrm{N})$ required for good reception. The $\mathrm{C} / \mathrm{N}$ is defined as:

$$
C / N=10 \cdot \log _{10}\left(\frac{P_{S}}{P_{N}}\right)
$$

where $P_{S}$ is the signal power and $P_{N}$ is the noise power.

The minimal signal to noise ratio $(\mathrm{C} / \mathrm{N})$ required for good reception is the smallest one that still provides acceptable reception. Firstly, only one transmitter may be present therefore one can observe the $\mathrm{C} / \mathrm{N}$ at which the displayed video starts to be corrupted; we have employed the emergence of blocks in the display for that purpose, since the appearing of blocks indicates a degradation of the video decoding indicating reception errors.

Another experiment (that occurs in continuation) encompasses varying the signal power difference (or ratio) between the signals arriving from the two "carriers" and searching for the same effect. This provides an estimate of the built-in receiver demodulation capability under inter-symbol interference. This was done for transmitter power ratios in the range from 0 to $21 \mathrm{~dB}$, in steps of $3 \mathrm{~dB}$. This is also evaluated for different relative delays in the range from 30 to $120 \mu \mathrm{s}$, in steps of $30 \mu \mathrm{s}$, and in the range from 120 to $200 \mu \mathrm{s}$, in steps of $10 \mu \mathrm{s}$. For each relative delay and power difference setting, the minimal $\mathrm{C} / \mathrm{N}$ required for accurate reception is measured, for each of the televisions sets available in laboratory.

Besides the obvious evaluation of the signal quality requirements for SFN deployment (in terms of required strength and maximal interference), the above discussed strategy provides a manner to evaluate and compare the performance of commercial DTV sets.

The experiments, its aspects and results concerning the diagram in Figure 2 are presented in Section IV.

\section{Equipment}

To implement the experiments illustrated in Figures 1 and 2, different equipment were necessary. For completeness, we present and describe the ones employed.

1) Exciter: it receivers the ASI streams from a multiplexer, timing, and frequency references of $1 \mathrm{pps}$ and $10 \mathrm{MHz}$, respectively, and generates the OFDM signal to be transmitted by the DTV broadcasting network. We employed two exciters (TX1 and TX2 in Figure 1 and Figure 2) from Hitachi Linear IS7001 in order to build the SFN, they are show in Figure 3;

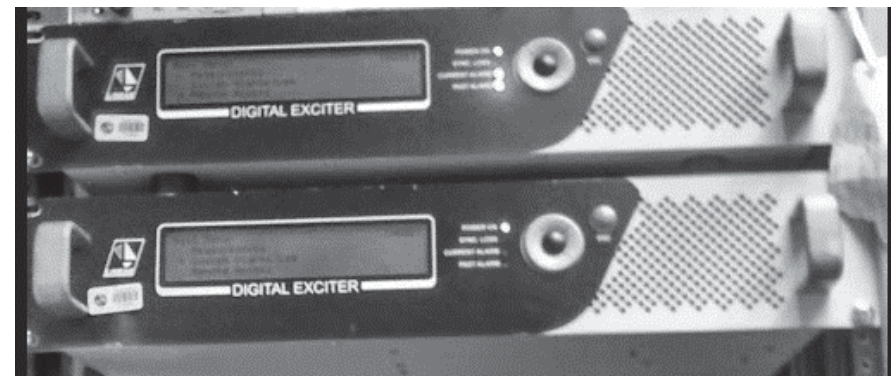

Figure 3. Hitachi Linear IS7001 Exciters Used in the Experiments.

2) Clock: an SFN requires precise timing and frequency references, i.e., "a master clock" [1]. We employ an EC22S Epsilon Clock GPS to produce those references using Global Positioning System signals. The EC225 is shown in Figure 4, in the diagrams in Figure 1 and Figure 2 it is denoted by the acronym GPS; 


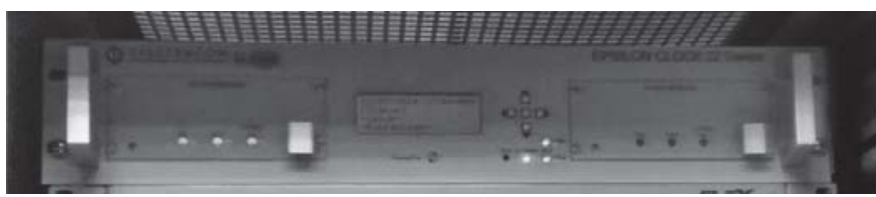

Figure 4. EC225 GPS clock and frequency references generator.

3) Multiplexer: in the diagrams in Figure 1 and Figure 2, the exciters are fed with the stream to be transmitted (TS). The BTS is produced by a multiplexer from NEC, model MX1500 , with the aid of the mux controller (MCT) which produces network and stream configuration paramenters;

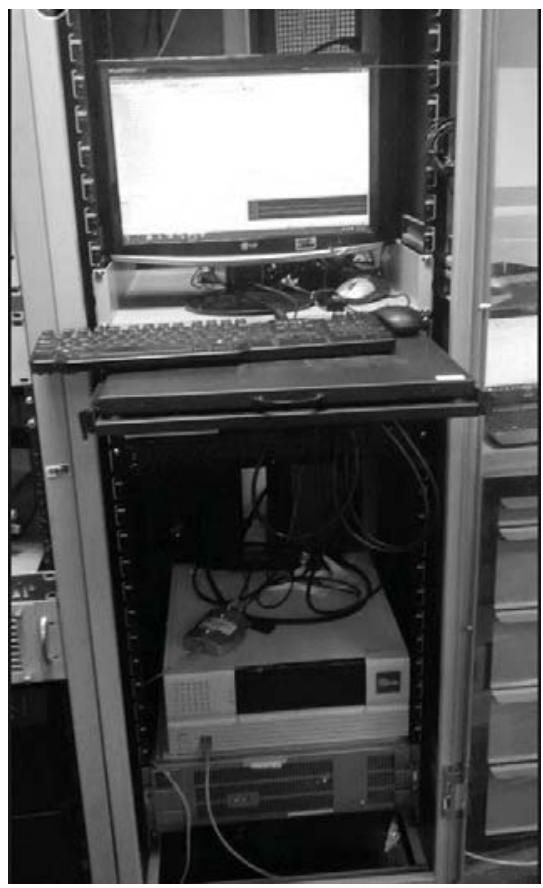

Figure 5. Picture of the Multiplexer and MCT used in the experiments.

4) Noise Generator: a "test transmitter" is used to generate pass-band white noise in order to simulate real transmission conditions. We employed a Rohde \& Schwarz SFE100 for that purpose, this is shown in Figure 6;

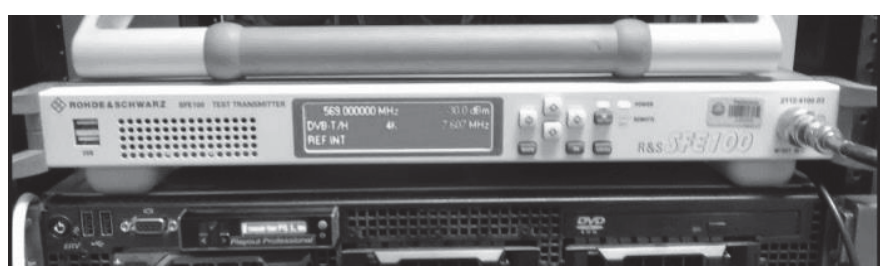

Figure 6. Rohde \& Schwarz SFE100 Test Transmitter used to generate noise and mimic real transmission conditions in the experiments.

5) TV Analyzer: the multipath OFDM signal produced using the experimental setup in Figure 1 is analyzed using a ISDBT signal analyzer, a Rohde \& Schwarz ETH TV analyzer was employed;
6) DTV Receivers: a series of displays/TV sets from different manufactures (produced between 2012 and 2013) were used in the experiments corresponding to Figure 2; the TV sets are presented in Figure 7.

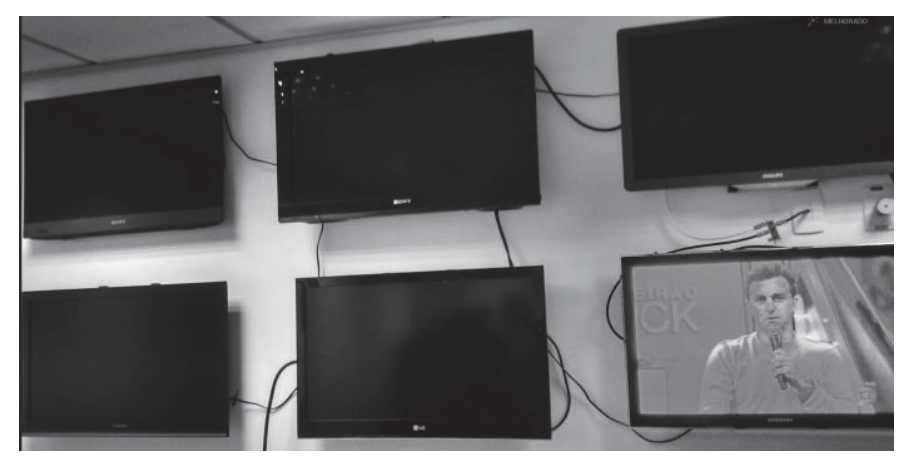

Figure 7. TV sets used in the experiments.

\section{CONSTELLATION BEHAVIOR: RESUlTS AND ANALYSIS}

If a single electromagnetic wave from only one transmitter arrives at a receiver, without any channel distortion, then it is easy for the receiver circuitry to estimate the in-phase and quadrature components of the symbols carried by each OFDM frequency carrier. Figure 8 illustrates the symbol constellation for the 64 QAM (64 symbols Quadrature Amplitude Modulation [5] set of possible symbols. There are 64 different symbols meaning that each 6 bits are carried by a symbol in each OFDM carrier. This constellation is obtained by applying a TV analyzer to the received SBTVD signal in order to analyze a carrier of the layer-B of the OFDM (SBTVD [6]) broadcast signal using 64 QAM. Some amplitude and phase spreads can be observed at the received signal that are due to different channel distortions and inherent system noise.

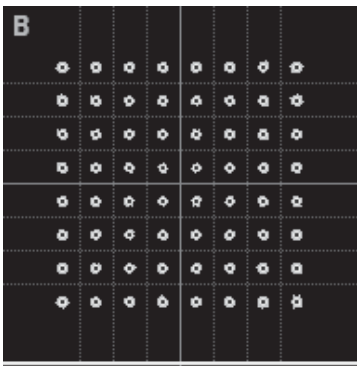

Figure 8. Received constellation of the 64 QAM in an OFDM carrier in the layer-B of the SBTVD signal.

As previously mentioned, the aim of the first experimental setup (the diagram in Figure 1) is to evaluate how the different power ratios and relative delays between a signal (a primary signal) and a "copy" of itself (the secondary signal) affect the reception in SFNs. Therefore, we analyzed the constellations that are obtained for some power ratios (or differences in $\mathrm{dB}$ ) and some relative delays or time alignment of the signals in the receiver input. This is intended trying to evaluate the reception in some usual and possible SFN scenarios. Figures 9, 10, 11, 12 and 13 show the behavior observed in laboratory for the 64 QAM constellation for increasing power ratios between the two signals. The symbol distributions in Figure 9 are obtained for signals arriving at the receiver with the same power while the ones in Figure 13 are obtained for the primary signal having a power that is 20 
$\mathrm{dB}$ greater than the secondary one. Each of these 5 figures present 11 constellations. Each of those 11 constellation in each figure corresponds to a different relative delay between the primary and secondary signals at the receiver.

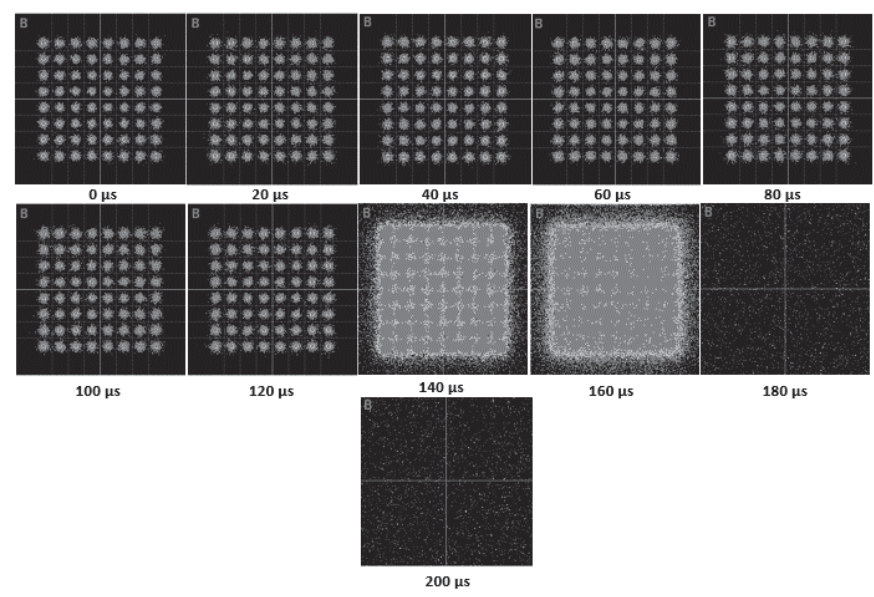

Figure 9. Received constellation of the 64 QAM in an OFDM carrier in the layer-B of the SBTVD signal when two signals of equal power are received for different relative delays between the received signals.
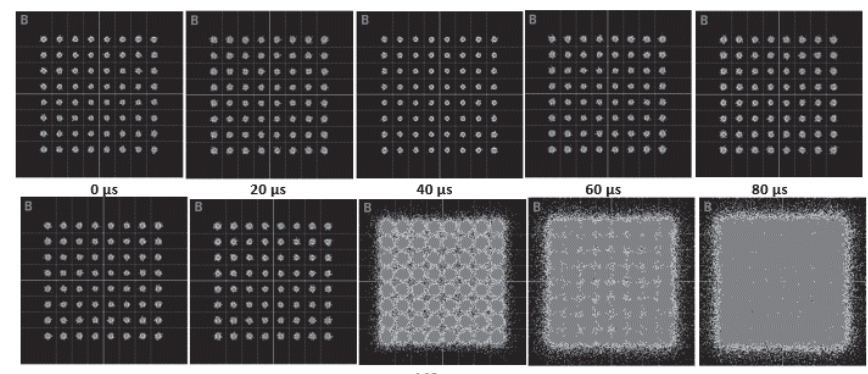

100 us

$120 \mu \mathrm{s}$

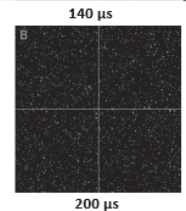

160 นs

180 นs

$200 \mu \mathrm{s}$

Figure 10. Received constellation of the 64 QAM in an OFDM carrier in the layer-B of the SBTVD signal when two signals having a power level difference of $5 \mathrm{~dB}$ are received for different relative delays between the received signals.
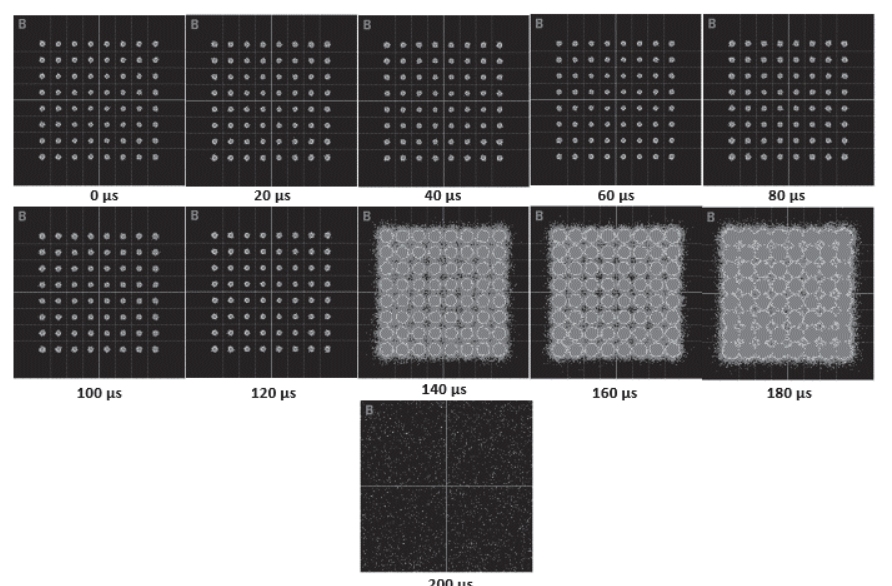

180

$160 \mu \mathrm{s}$

$200 \mu \mathrm{s}$

Figure 11. Received constellation of the 64 QAM in an
OFDM carrier in the layer-B of the SBTVD signal when two signals having a power level difference of $10 \mathrm{~dB}$ are received for different relative delays between the received signals.

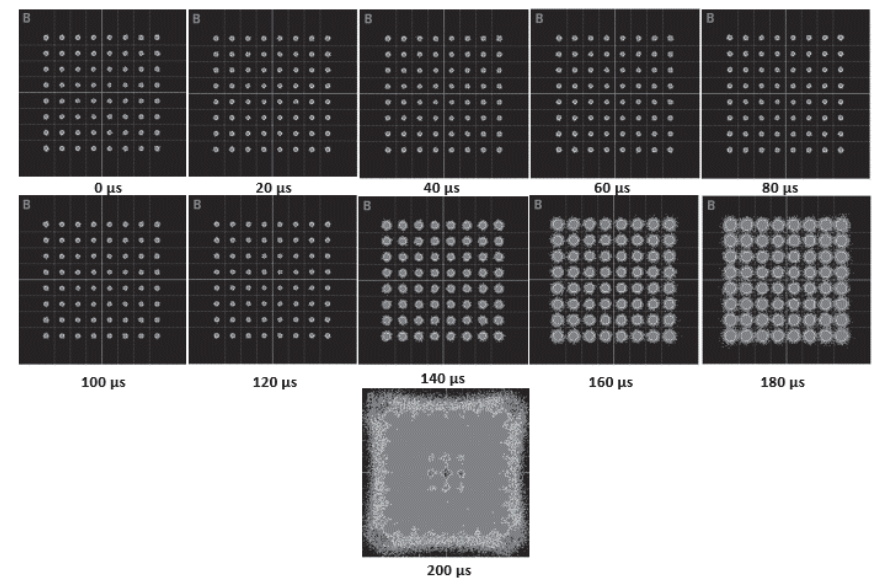

Figure 12. Received constellation of the 64 QAM in an OFDM carrier in the layer-B of the SBTVD signal when two signals having a power level difference of $15 \mathrm{~dB}$ are received for different relative delays between the received signals.

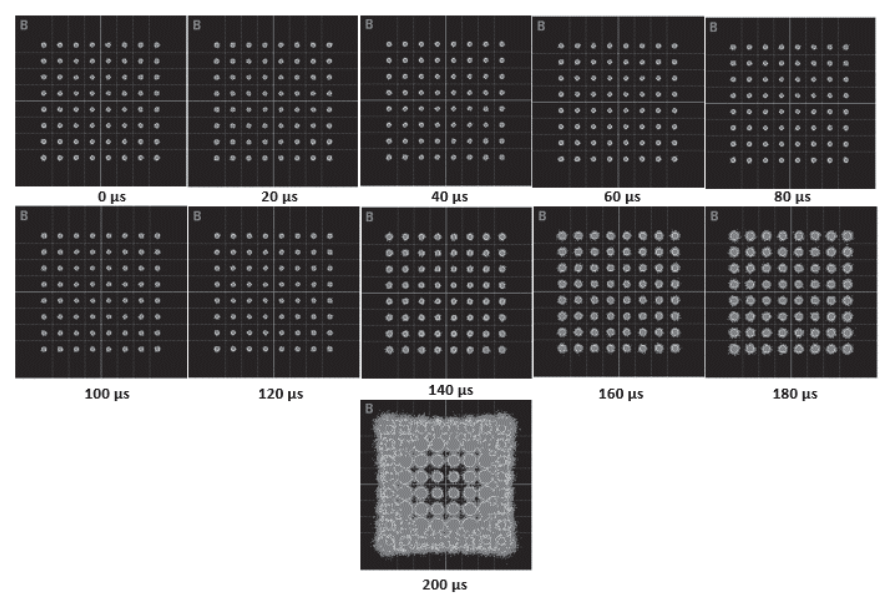

Figure 13. Received constellation of the 64 QAM in an OFDM carrier in the layer-B of the SBTVD signal when two signals having a power level difference of $20 \mathrm{~dB}$ are received for different relative delays between the received signals.

Figure 8 shows the effects of symbol recovery at the receiver that are already seen in the laboratory, i.e., even under controlled conditions. If carefully analyzed, it is observed that the symbols' recovery becomes harder as the symbol energy increases, i.e., as the symbol is more far from the origin of the IxQ plane. This is most probably due to the fact that the larger the amplitude of the components are, the more severe are the effects of circuitry non-linearities. The consequence is an increase on the symbol dispersion at the receiver as the symbol energy increases. This effect is also observable in the plots in Figure 9 to Figure 13 and it is further explained in Figure 14. Figure 14 presents the results and analysis on symbol dispersion presented in [3]. These show that symbol dispersion remarkably increases at the constellation symbols having greater energy levels, the outer ones of constellation symbols, what may hinder the signal's 
detection as the symbol's energy increases.

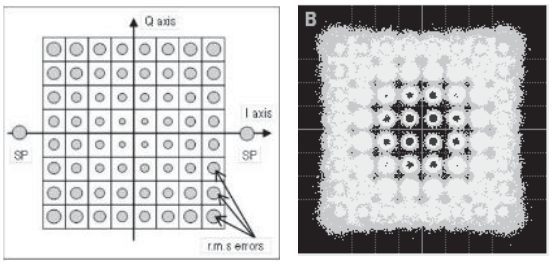

Figure 14. ITU study on the expected constellation behavior in a case of large constellation dispersion [3].

Observing the symbol constellations in Figure 9 to Figure 13, something that pops-up is that is that as the power difference between primary and secondary signals decrease, the dispersions of constellation symbols enlarge. For example, if one analyzes the case of the relative delay being $120 \mu \mathrm{s}$, for a difference of $0 \mathrm{~dB}$ between the signals, the dispersion of the symbol is much larger than for differences of 5, 10, 15 and $20 \mathrm{~dB}$. Actually, one readily sees that the symbol dispersions reduces as the power ratio increases.

From Figures 9, 10, 11, 12 and 13, one can also see that the dispersion of the symbols increases as the relative delay between the two signals increases, independently of their relative powers. We have also empirically observed that as soon as the delay surpasses $126 \mu$ s the dispersion drastically jumps up. This is an effect of the OFDM symbol guard interval [4]. Until that relative delay, reception under multipath condition (primary plus secondary signals) is achieved. Above that value, reception is not possible anymore in the worse conditions, i.e., small power level difference between primary and secondary signals.

Combining the above analyses (delay and power ratio influences on constellation dispersion), on notes that the influence or even the presence of the secondary signal can be disregarded if the difference between the power of the transmitters is larger than $15 \mathrm{~dB}$. That derives from the fact that in these cases the secondary signal is too small as compared to the primary one, resulting that it can be considered to be negligible, not imperiling (at least in the constellation analyzer) symbol detection. It shall be said that the behavior observed in the laboratory, which are illustrated in Figures 9, 10, 11, 12 and 13 are in consonance with the results and analysis reported in [3]. However, what is the real impact of SFN on actual DTV sets and in more realistic reception scenarios where noise is present?

\section{DTV SIGNAL RECEPTION IN A SiNGLE FREQUENCY NETWORK: RESULTS AND ANALYSIS}

\section{A. Undertaken Measures}

After evaluating how the received signal copies relative time alignment and power ratio influence reception, typical of an SFN, of the 64 QAM symbol constellation, we must move forward and evaluate the influence of the receiving conditions on actual commercial TV sets. For that purpose, five different TV sets were tried out as receivers in an SFN with two transmitters according to the diagram in Figure 2.

Before proceeding to receiver evaluation under SFN conditions, we measure the receiver performance for a non- distorting channel, that is, we evaluate the receiver's sensibility [6]. We measure the minimum signal power that guarantees correct reception of the OFDM symbols, this is done for each TV set. For that purpose one simply observes the decoded video and evaluates if there are blocks freezing effects (i.e., effects that would be perceived by common nonspecialist viewers). The last column at Table 2 presents these power levels for the different TV sets. We present them as required input power levels, as one feds the TV set input directly, ignoring the antenna although assuming impedance matching. For sake of non-disclosure of the TV sets brands they are represented by capital letters A to E. Table 2 presents in the second column the carrier to noise ratio $C / N_{\min }$ required for correct reception. The $[\mathrm{C} / \mathrm{N}]$ is measured assuming a received signal power of $-40 \mathrm{dBm}$ and the added noise power (see Figure 2) is varied so that the minimal $\mathrm{C} / \mathrm{N}$ for the one transmitter case is obtained, which is the simple difference between the $\mathrm{C}(\mathrm{dBm})$ and $\mathrm{N}(\mathrm{dBm})$ (thus $\mathrm{C} / \mathrm{N}$ is given in $\mathrm{dB}$ ).

TABLE 2

MINIMUM C/N AND MINIMUM INPUT POWER FOR CORRECT SYMBOL RECEPTION

\begin{tabular}{|c|c|c|}
\hline TV Set & $\begin{array}{c}\text { Input } \mathrm{C} / \mathrm{N}_{\text {mín }} \\
(\mathrm{dB})\end{array}$ & $\begin{array}{c}\text { Required Input } \\
\text { Power }\left(C_{\text {min }}\right)(\mathrm{dBm})\end{array}$ \\
\hline $\mathrm{A}$ & 17.8 & -80.6 \\
\hline $\mathrm{B}$ & 17.8 & -81.1 \\
\hline $\mathrm{C}$ & 16.7 & -83.2 \\
\hline $\mathrm{D}$ & 16.7 & -82.2 \\
\hline $\mathrm{E}$ & 16.7 & -84.8 \\
\hline
\end{tabular}

\section{B. Receiver Characteristics in an SFN Scenario}

So far, we have conducted and presented results for two different experiments with TV sets that evaluate their receiving performances for the single transmitter case. Now we advance to evaluate TV set receiver performances in an SFN scenario. For that, we consider an SFN composed of two transmitters as in Figure 2. The most basic objective is to obtain the minimum $\mathrm{C} / \mathrm{N}$ for different reception conditions. The primary transmitter power (P.Tx1) is set at $-40 \mathrm{dBm}$ while the second one (P.Tx2) is varied

$$
\begin{gathered}
D L[\mathrm{~dB}]=P . T x 1[\mathrm{dBm}]-P . T x 2[\mathrm{dBm}]= \\
-40[\mathrm{dBm}]-P . T x 2[\mathrm{dBm}] .
\end{gathered}
$$

Another aspect that is changed is the time alignment / relative delay between the two SFN signals at the receiver input. The SFN is configured to work (as in Table I) with a Guard Interval (GI) of $126 \mu$ s (1/8 of the OFDM symbol duration). For each combination of different power level from the primary to the secondary signal and relative delay between them, we evaluate the minimum value of $\mathrm{C} / \mathrm{N}$ that guarantees an acceptable reception. Table 3 to 7 present the measured $\mathrm{C} / \mathrm{N}_{\min }$ for the combinations of relative power and relative delay tested. In these tables, an " $\mathrm{X}$ " denotes that reception was impossible for the correspondent combination of power level difference and relative signal alignment between primary and secondary signals. In Tables 3 to 7 , the $\mathrm{C} / \mathrm{N}$ is defined as 


$$
C / N_{\min }=\frac{\operatorname{Pow}_{T \times 1+T \times 2}}{N_{\max }}
$$

as it is impossible to separate the two carriers in the TV set input in the present scenario.

TABLE 3

MINIMUM C/N GUARANTEEING ACCEPTABLE SIGNAL DECODING FOR THE TV SET A

\begin{tabular}{|c|c|c|c|c|c|c|c|c|}
\hline \multicolumn{9}{|c|}{$\begin{array}{c}\text { Minimum } \mathbf{C} / \mathbf{N} \text { for different configurations of level and relative delay } \\
\text { relations between carriers (dB) }\end{array}$} \\
\hline & \multicolumn{8}{|c|}{ 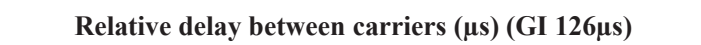 } \\
\hline $\begin{array}{c}\text { Diff. } \\
\text { Lv.(dB) }\end{array}$ & 30 & 60 & 90 & 120 & 130 & 150 & 170 & 200 \\
\hline $\mathbf{0}$ & 23 & 25.5 & 24.7 & 24 & $\mathrm{X}$ & $\mathrm{X}$ & $\mathrm{X}$ & $\mathrm{X}$ \\
\hline 3 & 20.6 & 21.6 & 20.6 & 20.6 & $\mathrm{X}$ & $\mathrm{X}$ & $\mathrm{X}$ & $\mathrm{X}$ \\
\hline 6 & 18.8 & 20.8 & 19.8 & 19.8 & $\mathrm{X}$ & $\mathrm{X}$ & $\mathrm{X}$ & $\mathrm{X}$ \\
\hline 9 & 18.2 & 18.2 & 18.2 & 18.2 & 23.9 & $\mathrm{X}$ & 21.2 & 25.7 \\
\hline 12 & 17.9 & 17.9 & 17.9 & 17.9 & 18.9 & 19.9 & 19.9 & 20.9 \\
\hline 15 & 17.8 & 17.8 & 17.9 & 17.8 & 18.8 & 19.8 & 19.8 & 20.8 \\
\hline 18 & 17.8 & 17.8 & 17.8 & 17.8 & 17.8 & 19.8 & 19.8 & 20.8 \\
\hline 21 & 17.8 & 17.8 & 17.8 & 17.8 & 17.8 & 17.8 & 17.8 & 18.8 \\
\hline
\end{tabular}

TABLE 4

MINIMUM C/N GUARANTEEING ACCEPTABLE SIGNAL DECODING FOR THE TV SET B

\begin{tabular}{|c|c|c|c|c|c|c|c|c|}
\hline \multicolumn{9}{|c|}{$\begin{array}{c}\text { Minimum } \mathbf{C} / \mathbf{N} \text { for different configurations of level and relative delay } \\
\text { relations between carriers (dB) }\end{array}$} \\
\hline & \multicolumn{8}{|c|}{ Relative delay between carriers ( $\mu$ s) (GI 126 $\mu$ s) } \\
\hline $\begin{array}{l}\text { Diff. } \\
\operatorname{Lv}(d B)\end{array}$ & 30 & 60 & 90 & 120 & 130 & 150 & 170 & 200 \\
\hline $\mathbf{0}$ & 23 & 23 & 23 & 23 & $\mathrm{X}$ & $\mathrm{X}$ & $\mathrm{X}$ & $\mathrm{X}$ \\
\hline 3 & 20.6 & 20.6 & 21.6 & 21.6 & 21.6 & 21.6 & 21.6 & 21.6 \\
\hline 6 & 19.8 & 19.8 & 19.8 & 19.8 & 19.8 & 19.8 & 19.8 & 19.8 \\
\hline 9 & 18.2 & 18.2 & 18.2 & 18.2 & 18.2 & 19.2 & 19.2 & 19.2 \\
\hline 12 & 17.9 & 17.9 & 17.9 & 17.9 & 17.9 & 17.9 & 17.9 & 17.9 \\
\hline 15 & 17.8 & 17.8 & 17.8 & 17.8 & 17.8 & 17.8 & 17.8 & 17.8 \\
\hline 18 & 17.8 & 17.8 & 17.8 & 17.8 & 17.8 & 17.8 & 17.8 & 17.8 \\
\hline 21 & 17.8 & 17.8 & 17.8 & 17.8 & 17.8 & 17.8 & 17.8 & 17.8 \\
\hline
\end{tabular}

TABLE 5

MINIMUM C/N GUARANTEEING ACCEPTABLE SIGNAL DECODING FOR THE TV SET C

Minimum $\mathbf{C} / \mathrm{N}$ for different configurations of level and relative delay relations between carriers $(\mathrm{dB})$

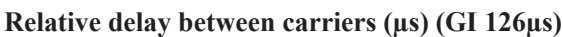

\begin{tabular}{|c|c|c|c|c|c|c|c|c|}
$\begin{array}{c}\text { Diff. } \\
\text { Lv.(dB) }\end{array}$ & $\mathbf{3 0}$ & $\mathbf{6 0}$ & $\mathbf{9 0}$ & $\mathbf{1 2 0}$ & $\mathbf{1 3 0}$ & $\mathbf{1 5 0}$ & $\mathbf{1 7 0}$ & $\mathbf{2 0 0}$ \\
\hline $\mathbf{0}$ & 23 & 23 & 23 & 23 & $\mathrm{X}$ & $\mathrm{X}$ & $\mathrm{X}$ & $\mathrm{X}$ \\
\hline $\mathbf{3}$ & 20.6 & 20.6 & 20.6 & 20.6 & 20.6 & 20.6 & 21.6 & 21.6 \\
\hline $\mathbf{6}$ & 18.8 & 18.8 & 18.8 & 18.8 & 18.8 & 18.8 & 19.8 & 19.8 \\
\hline $\mathbf{9}$ & 18.2 & 18.2 & 18.2 & 18.2 & 18.2 & 18.2 & 18.2 & 18.2 \\
\hline $\mathbf{1 2}$ & 17.9 & 17.9 & 17.9 & 17.9 & 17.9 & 17.9 & 17.9 & 17.9 \\
\hline $\mathbf{1 5}$ & 16.7 & 16.7 & 17.8 & 17.8 & 17.8 & 17.8 & 17.8 & 17.8 \\
\hline $\mathbf{1 8}$ & 16.7 & 16.7 & 17.8 & 17.8 & 17.8 & 17.8 & 17.8 & 17.8 \\
\hline $\mathbf{2 1}$ & 16.7 & 16.7 & 16.7 & 16.7 & 16.7 & 16.7 & 17.8 & 17.8 \\
\hline
\end{tabular}

TABLE 6

MINIMUM C/N GUARANTEEING ACCEPTABLE SIGNAL DECODING FOR THE TV SET D

Minimum $\mathbf{C} / \mathbf{N}$ for different configurations of level and relative delay relations between carriers $(\mathrm{dB})$

Relative delay between carriers ( $\mu \mathrm{s})$ (GI 126 $\mu \mathrm{s})$

\begin{tabular}{|c|c|c|c|c|c|c|c|c|}
\hline $\begin{array}{c}\text { Diff. } \\
\text { Lv.(dB) }\end{array}$ & $\mathbf{3 0}$ & $\mathbf{6 0}$ & $\mathbf{9 0}$ & $\mathbf{1 2 0}$ & $\mathbf{1 3 0}$ & $\mathbf{1 5 0}$ & $\mathbf{1 7 0}$ & $\mathbf{2 0 0}$ \\
\hline $\mathbf{0}$ & 22 & 22 & 22 & 23 & $\mathrm{X}$ & $\mathrm{X}$ & $\mathrm{X}$ & $\mathrm{X}$ \\
\hline $\mathbf{3}$ & 19.6 & 19.6 & 19.6 & 19.6 & 20.6 & 20.6 & 20.6 & 20.6 \\
\hline $\mathbf{6}$ & 18.8 & 18.8 & 18.8 & 18.8 & 19.8 & 19.8 & 19.8 & 19.8 \\
\hline $\mathbf{9}$ & 18.2 & 18.2 & 18.2 & 18.2 & 19.2 & 19.2 & 19.2 & 19.2 \\
\hline $\mathbf{1 2}$ & 17.9 & 17.9 & 17.9 & 17.9 & 17.9 & 17.9 & 17.9 & 17.9 \\
\hline $\mathbf{1 5}$ & 17.8 & 17.8 & 17.8 & 17.8 & 17.8 & 17.8 & 17.8 & 17.8 \\
\hline $\mathbf{1 8}$ & 17.8 & 17.8 & 17.8 & 17.8 & 16.7 & 16.7 & 16.7 & 17.8 \\
\hline $\mathbf{2 1}$ & 17.8 & 16.7 & 17.8 & 16.7 & 16.7 & 16.7 & 16.7 & 16.7 \\
\hline
\end{tabular}

TABLE 7

MINIMUM C/N GUARANTEEING ACCEPTABLE SIGNAL DECODING FOR THE TV SET E

\begin{tabular}{|c|c|c|c|c|c|c|c|c|}
\hline \multicolumn{9}{|c|}{$\begin{array}{c}\text { Minimum } \mathrm{C} / \mathrm{N} \text { for different configurations of level and relative delay } \\
\text { relations between carriers }(\mathrm{dB})\end{array}$} \\
\hline & \multicolumn{8}{|c|}{ 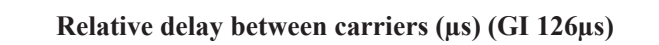 } \\
\hline $\begin{array}{c}\text { Diff. } \\
\text { Lv.(dB) }\end{array}$ & 30 & 60 & 90 & 120 & 130 & 150 & 170 & 200 \\
\hline $\mathbf{0}$ & 22 & 22 & 22 & 22 & 25.5 & $\mathrm{X}$ & $\mathrm{X}$ & $\mathrm{X}$ \\
\hline 3 & 19.6 & 19.6 & 19.6 & 20.6 & 21.6 & 24.1 & 23.9 & 24.9 \\
\hline 6 & 18.8 & 18.8 & 18.8 & 19.8 & 19.8 & 19.8 & 19.8 & 19.8 \\
\hline 9 & 18.2 & 18.2 & 18.2 & 18.2 & 18.2 & 18.2 & 18.2 & 18.2 \\
\hline 12 & 17.9 & 17.9 & 17.9 & 17.9 & 17.9 & 17.9 & 17.9 & 17.9 \\
\hline 15 & 16.7 & 16.7 & 17.8 & 17.8 & 17.8 & 17.8 & 17.8 & 17.8 \\
\hline 18 & 16.7 & 16.7 & 17.8 & 17.8 & 17.8 & 17.8 & 17.8 & 17.8 \\
\hline 21 & 16.7 & 16.7 & 17.8 & 17.8 & 17.8 & 17.8 & 17.8 & 17.8 \\
\hline
\end{tabular}


All TV sets provided an acceptable reception at both signal arriving at it with the same power level, i.e, the TV sets were able to demodulate the received signal, as far as the time shift between the signals is smaller than the OFDM guard interval; TV set $\mathrm{E}$ that could do that even for a relative delay a bit larger than the guard interval. Almost all TV sets evaluated were capable to receive the signal in all the remaining cases when the power levels difference between primary and secondary waves larger than $0 \mathrm{~dB}$; the was the TV set A. In addition, from the results one also observes the reduction of the required $\mathrm{C} / \mathrm{N}$ as the power level difference between the two SFN signals increases.

\section{Margin Loss Due to SFN}

We investigate now if there is any difference in receiver performance between the cases when there is only one signal copy arriving at the receiver input and when there are multiple copies. A simple comparison between the entries in Table 2 and the ones in Tables 3 to 7 shows that in the SFN scenario the required $\mathrm{C} / \mathrm{N}$ for acceptable reception is larger than the one that is required in the single arriving signal scenario. That is, there is a reception margin loss $[3,4]$ when the TV broadcast network shifts from an MFN paradigm to the SFN paradigm. This margin loss can be computed by means of

$$
\begin{aligned}
\operatorname{Margin} \operatorname{Loss}(d B)= & C / N_{\min .1 T x}(d B) \\
& -C / N_{\text {min.SFN }}(d B)
\end{aligned}
$$

Table 8 presents the SFN margin losses in the worst-case scenario, the most demanding one in terms of the $\mathrm{C} / \mathrm{N}$ required for acceptable reception in the two-transmitter SFN case, for each of the five tested TV receivers. In the second column of Table 8 contains the SFN margin loss when the secondary signal arrives within the OFDM guard interval (for exception of TV set A) the margin loss when the secondary signal arrives after the guard interval.

TABLE 8

C/N MARGIM LOSS IN AN SFN FOR DIFFERENT TV SETS

\begin{tabular}{|c|c|c|}
\hline Model & Inside GI & Outside GI \\
\hline A & 5 & 9 \\
\hline B & 5 & 3 \\
\hline C & 6 & 4 \\
\hline D & 4 & 4 \\
\hline E & 5 & 7 \\
\hline
\end{tabular}

From the simple average of the values in Table 8 , we averaged the SFN's margin loss for commercial receptors (Table 9).

TABLE 9

LOSS OF C/N MARGIN DUE THE PRESENCE OF A SECOND SIGNAL IN THE SFN

\begin{tabular}{|c|c|}
\hline \multicolumn{2}{|c|}{ Loss of $\mathrm{C} / \mathrm{N}$ margin $(\mathrm{dB})$} \\
\hline Inside $\mathrm{Gl}$ & 5 \\
\hline Outside $\mathrm{Gl}$ & 5.4 \\
\hline
\end{tabular}

The TV sets tested could not demodulate the signal, except for television $\mathrm{E}$, once the relative delay between the signal surpassed the GI (126 $\mu \mathrm{s})$ and for a difference level of $0 \mathrm{~dB}$ between the two signals arriving at the receiver. However, in all other cases (for exception of TV set A) it was possible to correctly recover the signal in all other conditions even with the decrease of $\mathrm{C} / \mathrm{N}$.

\section{MINIMUM FIELD STRENGTH FOR RECEPTION IN AN SFN}

The use of SFN instead of MFN broadcasting provokes a reception margin loss, meaning that a larger $\mathrm{C} / \mathrm{N}$ is required for reception than in an SFN. Tables 3, 4, 5, 6 and 7 present the minimum $\mathrm{C} / \mathrm{N}$ values for each tested receiver in different reception conditions. These compared with the values in Table 2, provide the $\mathrm{C} / \mathrm{N}$ margin loss which can in turn be converted into the minimal signal power required for reception in an SFN, deriving from its multipath reception scenario.

\section{A. Calculation model}

To calculate the minimum electric field strength that reaches the reception antennas, considering only external antennas in SFN area, the factors pointed in Table 10 are partly related to external reception conditions (after the receiver) considered in the CPqD's Digital Channel Planning [7].

TABLE 10

RECEIVER MODEL USED TO OBTAIN FIELD STRENGTH FROM POWER

\begin{tabular}{|c|c|c|}
\hline Factor & Symbol & Value \\
\hline Bandwidth $(\mathrm{MHz})$ & $\mathrm{B}$ & 6 \\
\hline $\begin{array}{c}\text { Boltzmann constant } \\
(\mathrm{Ws} / \mathrm{K})\end{array}$ & $\mathrm{K}$ & $\begin{array}{c}1.38 \mathrm{E}- \\
23\end{array}$ \\
\hline Absolute temperature $(\mathrm{K})$ & $\mathrm{T}$ & 290 \\
\hline Thermal noise $(\mathrm{dBm})$ & $\mathrm{Nt}$ & -106.20 \\
\hline Central frequency $(\mathrm{MHz})$ & $\mathrm{fc}$ & 563 \\
\hline Carrier wavelength $(\mathrm{m})$ & 1 & 0.53 \\
\hline Effect area of isotropic antenna $\left(\mathrm{dBm}{ }^{2}\right)$ & $\mathrm{Ai}$ & -16.46 \\
\hline Half-wavelength dipole gain w.r.t the isotropic & $\mathrm{Gi}$ & 2.15 \\
\hline antenna $(\mathrm{dBi})$ & $\mathrm{G}$ & 10 \\
\hline Antenna gain w.r.t the half-wave dipole $(\mathrm{dBd})$ & $\mathrm{A}$ & -6.31 \\
\hline Effective area of antenna $(\mathrm{dBm} 2)$ & $\mathrm{h}$ & 377 \\
\hline Instrinsic impendance $(\Omega)$ & $\mathrm{Kd}$ & -130.07 \\
\hline Dipole fator $(\mathrm{dBm}-\mathrm{dB} \mu \mathrm{V} / \mathrm{m})$ & $\mathrm{Lf}$ & 4 \\
\hline Cable loss $(\mathrm{dB})$ & $\mathrm{Mm}$ & 0 \\
\hline Margin against man-made noise $(\mathrm{dB})$ & & \\
\hline
\end{tabular}

\section{B. MFN Reception Margin Loss}

Based on the values of Tables 3, 4, 5, 6 and 7, the minimum $\mathrm{C} / \mathrm{N}$ values, the receptor's noise figure land the SFN's multipath margin were measured, and, with Equation 5, the minimum signal power was calculated (Table 11).

$$
\begin{aligned}
P_{s}(d B m)=N_{t}(d B m) & +F_{r}(d B m)+C / N(d B) \\
& +M_{S F N}(d B)
\end{aligned}
$$

The SFN margin loss is given by the values in the fourth column of Table 8 , considering reception in proper conditions (inside the guard interval). These values are the increase in the minimum required signal power at the antenna (for correct reception) w.r.t. the single case scenario (MFN). 
In Equation (5) $N_{t}$ refers to the thermal noise, that will obviously be present. We consider it equal to the one provide by the external DTV reception conditions in [4], i.e. $N_{t}=$ $106.20 \mathrm{dBm}$ (temperature of $\mathrm{T}=290 \mathrm{~K}$, both of these are in Table 10). Fr refers to the receiver noise figure that is calculated using the minimum $\mathrm{C} / \mathrm{N}$, minimum input power $\left(C_{\text {min }}\right)$, being given by

$$
F_{r}(d B m)=C_{\min }(d B m)-C / N(d B)+N_{t}(d B m)
$$

TABLE 11

PERFORMANCES OF DIGITAL TV RECEIVERS EVALUATED

\begin{tabular}{|c|c|c|c|c|}
\hline \multirow{2}{*}{ Receiver } & $\begin{array}{c}\text { Digital } \\
\text { System } \\
\text { C/N } \\
\text { Threshold } \\
\text { (dB) }\end{array}$ & $\begin{array}{c}\text { Receiver's } \\
\text { Noise } \\
\text { Figure } \\
\text { (dB) }\end{array}$ & $\begin{array}{c}\text { SFN } \\
\text { Multipath } \\
\text { Margin } \\
\text { Loss(dB) }\end{array}$ & $\begin{array}{c}\text { Minimun } \\
\text { Signal } \\
\text { Power } \\
\text { (dBm) }\end{array}$ \\
\cline { 2 - 5 } & \multicolumn{5}{|c|}{ Symbol } \\
\cline { 2 - 6 } & C/N & Fr & Msfn & Ps \\
\hline A & 17.8 & 7.8 & 5.2 & -75.40 \\
\hline B & 17.8 & 7.3 & 5.2 & -75.90 \\
\hline C & 16.7 & 6.3 & 6.3 & -76.90 \\
\hline D & 16.7 & 6.3 & 4.2 & -79.00 \\
\hline E & 16.7 & 4.7 & 5.3 & -79.50 \\
\hline
\end{tabular}

\section{Minimum electric field strength for reception}

Above, we derived the required signal power for reception in an SFN (two-path reception). This is the necessary excitation at each receiver RF (Radio Frequency) input. However, this excitation will depend on several other factors as internal or external reception, antenna size, impedance matching, etc. That is, how is the propagated energy captured and input into the receiver RF input.

Using the reception model in Table 9 and the required signal power in Table 11, we can compute the minimum electric field strength arriving at the antenna that is required for correct reception of the DTV in an SFN network. The minimum field strength that shall arrive at the reception antenna can be calculated using [7]

$$
\begin{aligned}
E_{\min }(d B \mu V / m)= & P_{s}(d B m)+L_{f}(d B)+M_{m}(d B) \\
& -G(d B)-k_{d}(d B m \\
& -d B \mu V / m)
\end{aligned}
$$

For the evaluated DTV receivers the required field strength at the antenna (for the above model: Equation (7) and Table 10) are presented in Table 12, where one observes that they fit reasonably within $\mathrm{a}+-3 \mathrm{~dB}$ range.

\section{TABLE 12}

MINIMUM FIELD STRENGTH BY THE DTV RECEIVERS TESTED

\begin{tabular}{|c|c|}
\hline Receiver & $\begin{array}{c}\text { Minimum filed strength }\left(\mathbf{E}_{\mathbf{m i n}}\right) \\
(\mathbf{d B m})\end{array}$ \\
\hline $\mathrm{A}$ & 48.68 \\
\hline
\end{tabular}

\begin{tabular}{|c|c|}
\hline B & 48.18 \\
\hline C & 47.18 \\
\hline D & 45.08 \\
\hline E & 44.58 \\
\hline
\end{tabular}

\section{Correction factor for locations}

The values in Table 12 were obtained using a laboratory setup mimicking a two path reception scenario, which is a reasonable assumption for SFN DTV broadcasting. However, obviously in a real reception scenario the strength of the electric field may wander due to several factors (climatology, humidity, etc). Consequently, the field strength is assumed to vary with the considered location/site and time/date. Therefore, one must introduce a margin in the minimum required electric field strength to guarantee that the wave can be correctly received and decoded with acceptable quality, that is for the DTV broadcast being available for the receiver/viewer/consumer. Obviously, this margin increases as the resilience of the system to field strength variation rises, that is, as system availability increases.

$$
\Delta L(d B)=\mu \cdot \sigma m(d B) .
$$

Following the methodology in [7], we compute the correction factor (margin) as Equation (8). Again, as in [7], the employed $\Delta L$ is such that at $95 \%$ of the SFN coverage area the electric field meets the required electric field strength at least at $90 \%$ of the time. In this case, it is necessary to have a margin (apply a correction factor) $\Delta L$ that is computed considering a distribution $\mu$ of 1.64 times the standard deviation $\sigma m$ at large scale, presented in Figure 15. If one considers that the system has a large number of elements, then the latter is $5.5 \mathrm{~dB}$ [7]. Putting together, this results in a correction of the normal curve given by a factor, $\Delta L=1.64$. $5.5=9 \mathrm{~dB}$.

Each one of the tested DTV receivers can use the correction factor / margin above to compute the required field strength. Those are obtained by means of

$$
E_{\text {corrected }}(d B \mu V / m)=E_{\min }(d B \mu V / m)+\Delta L(d B)
$$

Which are presented in the last column in Table 12.

These values bring a worst-case scenario for the field strength of $58 \mathrm{~dB} \mu \mathrm{V} / \mathrm{m}$. Thus, when planning SFN DTV broadcasting the field strength must consider that in order for providing a reasonable DTV signal availability. It is worth noticing that our analysis produces an electrical field requirement that is $7 \mathrm{~dB}$ larger than the actual minimum field strength considered for DTV system deployment in Brazil (51 $\mathrm{dB} \mu \mathrm{V} / \mathrm{m})[7]$. 


\section{REFERENCES}

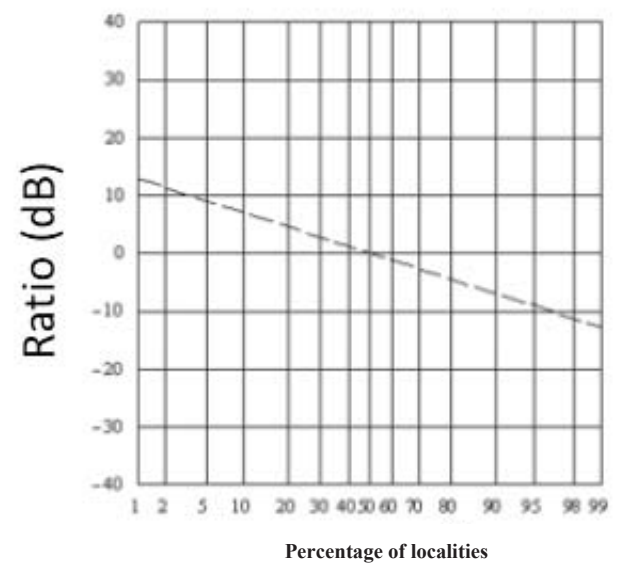

Figure 15. Field rate for a given percentage of time for a field for $50 \%$ of locations, also known as standard deviation at large scale $\sigma m$.

TABLE 13

MINIMUM PROPOSAL FIELD STRENGTH FOR SEVERAL DIGITAL TELEVISION RECEIVERS

\begin{tabular}{|c|c|}
\hline Receiver & $\begin{array}{c}\text { Minimum proposal field } \\
\text { strength }(\boldsymbol{d} \boldsymbol{B} \boldsymbol{\mu} \boldsymbol{~} \boldsymbol{m})\end{array}$ \\
\hline Samsung UN32D5500RGXZD & 57.7 \\
\hline Philips 32PFL7606D/78 & 57.2 \\
\hline LG 32CS460 & 56.2 \\
\hline Sony KDL-32EX525 & 54.1 \\
\hline Panasonic TC-L32X30B & 53.6 \\
\hline
\end{tabular}

\section{CONCLUSIONS}

The results Table 13 show the feasibility of SFN for the actual scenario and technology of DTV, but a slight performance variation between them. Another important aspect brought by these results is that it may be necessary to revise the minimum electric field strength for designing SFN DTV broadcast networks. When comparing them to the results presented in [7], there is the need for a $7 \mathrm{~dB}$ increment for the minimum electric field strength default value used in projects $(51 \mathrm{~dB} \mu \mathrm{V} / \mathrm{m})$ seems to be necessary. This increment may be required in general DTV coverage design, as SFN gets increasingly common. The minimum field strength value of $58 \mathrm{~dB} \mu \mathrm{V} / \mathrm{m}$ obtained in our analysis incorporated both the margin loss due to SFN usage and the $95 \%$ correction factor (according to international standards for digital television planning).
[1] Duca, Carolina, Yamada, Fujio. "Distribuição de sinais para o sistema Brasileiro de TV Digital”, Revista de Radiodifusão - SET, vol. 3, 2009.

[2] Associação Brasileira de Normas Técnicas, Televisão digital terrestre Sistema de transmissão, ABNT NBR 15601, Rio de Janeiro, 2008.

[3] International Telecommunications Union, "Report ITU-R BT.2209-1: Calculation model for SFN reception and reference receiver characteristics of ISDB-T system", Geneva, 2013.

[4] International Telecommunication Union, "Report ITU-R BT.1368-12: Planing criteria, including protection ratios, for digital terrestrial television services in the VHF/UHF bands," Geneva, 2015.

[5] Haykin, Simon, Communication Systems,,John Wiley \& Sons, 2001.

[6] Razavi Behzard, "RF Microeletronics", "Practice Hall", 2nd edition, 2012.

[7] Fundação CPqD - Centro de Pesquisa e Desenvolvimento em Telecomunicações. "Planejamento de Canais de TV Digital", São Paulo, 2003
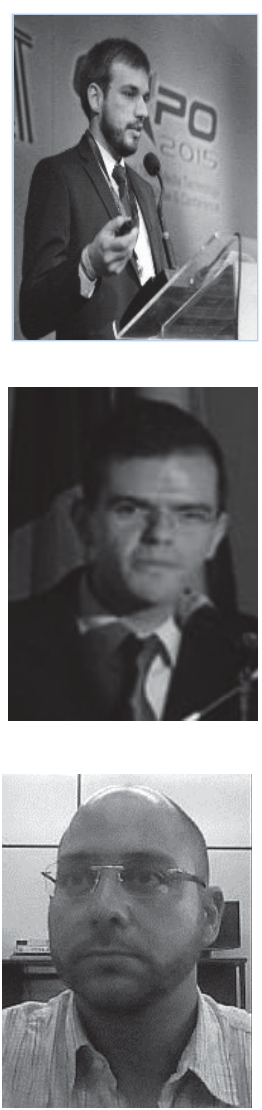

Chrisitan Fragoas Fernandez Rodrigues, master's student at Universidade do Estado do Rio de Janeiro, graduated in Systems Analysis Development at Centro Universitário da Cidade (2011) and Electrical Engineering with emphasis on Telecommunications at Universidade do Estado do Rio de Janeiro (2015). Is currently Project Engineer at TV Globo in digital television broadcasting area.

Francisco de Assis Peres graduated in Telecommunications Engineering from Universidade Federal Fluminense, completed his master's degree in Electromagnetics by Pontifícia Universidade Católica do Rio de Janeiro. Worked for 12 years as a Project Engineer at TV Globo drawing antennas and distributed systems for MFN and SFN systems. Currently manages the Department of Projects and Transmissions Systems Installations.

Lisandro Lovisolo earned a Phd in Electrical Engineering from Universidade Federal do Rio de Janeiro. Is currently professor in the Department of Eletronics and Telecommunications of Universidade do Estado do Rio de Janeiro. He is also part of the graduate program in Electronic Engineering of the institution. $\mathrm{He}$ founded the PROSAICO (Signal Processing Laboratory, Intelligent Applications and Communications, www.prosaico.uerj.br) and operates mainly in signals and communications processing areas. 